\title{
Relação de insegurança alimentar com condições de saúde materno-infantil
}

\author{
Élida Mara Braga Rocha ${ }^{1}$, Roberto Teixeira de Lima ${ }^{2}$ e Sophia Cornbluth Szarfarc ${ }^{3}$
}

O objetivo desse artigo foi associar a insegurança alimentar e nutricional (IA) com as condições de saúde materno-infantil. Trata-se de pesquisa transversal com crianças menores de cinco anos de idade, no Crato (CE), Brasil. A amostra foi probabilística com duas fases de seleção. No Dia Nacional da Multivacinação de 2010 foram coletados dados de 370 famílias com auxílio de um questionário semi-estruturado, abordando condições socioeconômicas e de saúde materno-infantil, além de utilizar a Escala Brasileira de Insegurança Alimentar. Entre as famílias atendidas pela Estratégia Saúde da Família 67,7\% estavam com algum grau de insegurança alimentar (IA). A falta de informações sobre alimentação infantil foi mais alta em famílias com IA (69\%). Quanto as morbidades, famílias em IA apresentaram maiores índices de crianças com diarreia $(O R=2,12$; IC95\%:1,03-4,38) e com febre e tosse $(O R=2,54$; IC95\%:1,28-5,04). Dessa forma, famílias em IA são atendidas pela ESF por serem mais vulneráveis socialmente, mas não recebem ações educativas sobre saúde infantil, podendo levar a doenças agudas e crônicas.

Palavras-chave: Brasil, educação alimentar e nutricional, pré-escolar, saúde materno-infantil, segurança alimentar e nutricional.

\section{Relation of food insecurity with health conditions of maternal and child}

The aim of this paper was to associate the food and nutrition insecurity (FI) with the conditions of maternal and child health. A cross-sectional survey with children under five years of age, in Crato (CE), Brazil. The sample was random with two-stage selection. National Day of Multivaccination 2010 data were collected from 370 families with the help of a semi-structured questionnaire, addressing socioeconomic conditions and maternal and child health, in addition to using the Brazilian Food Insecurity Scale. Among the families served by the Family Health Strategy $67.7 \%$ had some degree of food insecurity. The lack of information on infant feeding was highest in families FI $(69 \%)$. As morbidities family living in IA were more likely to have children with diarrhea (OR $=2.12$, IC95\%: 1.03-4.38), fever and cough (OR= 2.54, IC95\%:1.28-5.04). Thus, families in FI are met by ESF to be more socially vulnerable, but do not receive educational initiatives regarding child health and can lead to acute and chronic diseases.

Key-words: Brazil, food and nutrition education, preschool, maternal and child health, food security.

\footnotetext{
${ }^{1}$ Doutoranda, Pós-Graduação em Nutrição em Saúde Pública, Faculdade de Saúde Pública, Universidade de São Paulo, São Paulo, Brasil. Faculdade de Saúde Pública/USP. Correspondência: Av. Dr. Arnaldo no 715, CEP 01246-904 - Cerqueira César, São Paulo, SP. E-mail: elidamara@usp.br

${ }_{2}^{2}$ Professor, Pós-Graduação em Nutrição, Universidade Federal da Paraíba, João Pessoa, Brasil.

${ }^{3}$ Professora Associada do Departamento de Nutrição, Pós-Graduação em Nutrição em Saúde Pública, Faculdade de Saúde Pública, Universidade de São Paulo, São Paulo, Brasil.
} 


\section{INTRODUÇÃO}

A segurança alimentar e nutricional (SAN) tem como princípio a garantia de que todo ser humano tem o direito à alimentação digna, assegurando desse modo o bem-estar e a saúde do indivíduo [1]. Assim, a SAN está associada desde a satisfação psico-fisiológica até a alimentação saudável, sendo vista como um conjunto de direitos e políticas sociais contidas na noção de seguridade social. Desse modo os diversos aspectos que envolvem SAN, como o biológico, o econômico, o social, o ambiental e o cultural, permitem, reconhecer a pluralidade de seu conceito [2].

Dessa forma, a mensuração da SAN, em conjunto com outros indicadores de avaliação da saúde infantil, é uma iniciativa importante que viabiliza o acesso à qualidade de vida pela população.

Conforme observado por Habicht et al. [3] estudos que analisam a prevalência de insegurança alimentar em distintas populações do país são essenciais e decisivos para o planejamento de programas e políticas públicas, uma vez que possibilitam detectar as prioridades locais, possibilitando estabelecer intervenções específicas e direcionadas a assegurar o Direito Humano à Alimentação Adequada (DHAA).

Sendo assim, o objetivo desse artigo foi associar a situação de insegurança alimentar e nutricional com as condições de saúde materno-infantil no semiárido brasileiro.

\section{METODOLOGIA}

A pesquisa foi do tipo transversal com abordagem descritiva quantitativa e analítica. A população do estudo foi constituída por crianças menores de cinco anos de idade residentes no município de Crato (CE), que compareceram aos postos de saúde na segunda etapa da Campanha de Multivacinação, em Agosto de 2010.

A coleta de dados no Dia Nacional da Multivacinação é uma estratégia válida, que otimiza os custos financeiros, a logística e o tempo gasto na coleta de dados quando comparado a estudos dessa natureza realizados por meio de visitas domiciliares ${ }^{[4,5,6]}$.

O cálculo amostral foi baseado nas informações de monitoramento da imunização do ano de 2009 deste mesmo município [7, contando com a vacinação de cerca de 10.500 crianças menores de cinco anos de idade. Tomou-se por base a prevalência de 67,5\% de Insegurança Alimentar para crianças menores de cinco anos de idade no Nordeste, segundo a Pesquisa Nacional de Amostra Domiciliar (PNAD 2004) [8]. Adotou-se um erro amostral de $5 \%$ e um Intervalo de Confiança (IC) de 95\%, além do acréscimo de $10 \%$ para perda amostral, o que totalizou uma amostra de 360 crianças.

A amostragem foi do tipo probabilística, por conglomerado, sendo definida em duas fases de seleção. A primeira fase compreendeu o sorteio aleatório de seis das trinta Unidades Básicas de Saúde e a segunda fase constou de amostragem sistemática, na qual as crianças foram selecionadas na fila por ordem de chegada.

Foi utilizado um questionário socioeconômico pré-codificado direcionado aos pais das crianças para a caracterização da amostra (renda familiar per capita, escolaridade materna em anos completos concluídos, participação em programas sociais), por serem conhecidos como importantes condicionantes da insegurança alimentar.

A coleta de dados das condições de saúde infantil teve como foco: o atendimento pela Estratégia Saúde da Família (ESF), as visitas do Agente Comunitário de Saúde (ACS), além de acompanhamento pré-natal, o recebimento de suplemento de vitamina A e ferro e de informações sobre alimentação da criança e as ocorrências de doenças comuns da infância (sibilância, diarreia, febre e tosse), considerando como referência a Pesquisa Nacional de Demografia e Saúde, de 2006 [?].

Para diagnóstico da situação de segurança alimentar, a Escala Brasileira de Insegurança Alimentar (EBIA) validada para população brasileira, foi utilizada. Essa escala classifica as famílias em situação de segurança alimentar ( 0 pontos), insegurança alimentar leve (1-5 pontos), insegurança alimentar moderada (610 pontos) e insegurança alimentar grave (11-15 pontos) ${ }^{[10]}$.

As análises estatísticas foram realizadas com auxilio do software SPSS 14.0 for Windows. Em primeiro lugar, a amostra foi caracterizada por meio de análise descritiva. Em seguida a associação existente entre as variáveis selecionadas com a situação de IA foi realizada por meio de teste de Qui-Quadrado $\left(\chi^{2}\right)$, fixado $p<0,05$. Por fim, foi realizada uma análise 
bivariada entre IA e os fatores de risco e calculados os Odds Ratio (OR) e intervalos de confiança (IC95\%), considerando como significativo os valores de $p<0,05$.

A pesquisa foi previamente submetida à análise pelo Comitê de Ética em Pesquisa (CEP/HULW) da Universidade Federal da Paraiba, onde obteve aprovação para sua realização e publicação sob protocolo de no 346/10.

\section{RESULTADOS}

No Dia Nacional de Vacinação 370 responsáveis por crianças menores de cinco anos foram entrevistados. Os dados permitiram proporcionalidade de famílias residentes nas áreas urbana e rural já que aproximadamente $25 \%$ da amostra pertenciam à área rural. Foi observado que $40,7 \%$ das famílias apresentavam renda per capita menor que $1 / 4$ do salário mínimo o que está de acordo com a taxa de $48,4 \%$ das que recebiam benefício social. Mais de metade dos responsáveis pelas crianças tinham mais de nove anos de estudo (56,2\%). De acordo com a EBIA, a insegurança alimentar (IA) estava presente entre 58,1\% das famílias.

Tabela 1. Análise univariada, situação de insegurança alimentar segundo assistência em saúde e morbidades infantis, Crato (CE), Brasil, 2010.

\begin{tabular}{|c|c|c|c|c|c|}
\hline \multirow{3}{*}{ Variáveis } & & & \multicolumn{3}{|c|}{ Insegurança Alimentar } \\
\hline & \multicolumn{2}{|c|}{ Geral } & \multirow{2}{*}{$\begin{array}{c}\text { Leve } \\
\% \\
\end{array}$} & \multirow{2}{*}{$\begin{array}{c}\text { Moderada } \\
\% \\
\end{array}$} & \multirow{2}{*}{$\begin{array}{c}\text { Grave } \\
\% \\
\end{array}$} \\
\hline & $\mathbf{n}$ & $\%$ & & & \\
\hline \multicolumn{6}{|l|}{ Assistência em saúde } \\
\hline \multicolumn{6}{|l|}{ Atendido pela ESF $* *$} \\
\hline $\operatorname{Sim}$ & 211 & 57,0 & 39,3 & 19,4 & 9,0 \\
\hline Não & 159 & 43,0 & 25,2 & 15,7 & 4,4 \\
\hline \multicolumn{6}{|l|}{ Visita do ACS } \\
\hline $\operatorname{Sim}$ & 228 & 61,6 & 37,7 & 18,4 & 7,0 \\
\hline Não & 142 & 38,4 & 26,1 & 16,9 & 7,0 \\
\hline \multicolumn{6}{|c|}{ Trimestre de início do pré-natal * } \\
\hline 10 & 308 & 88,5 & 32,1 & 16,9 & 6,5 \\
\hline $2 \underline{o}-3 \underline{o}$ & 40 & 11,5 & 40,0 & 25,0 & 15,0 \\
\hline \multicolumn{6}{|l|}{ № de consultas de pré-natal * } \\
\hline$<6$ & 42 & 13,3 & 45,2 & 26,2 & 4,8 \\
\hline$\geq 6$ & 273 & 86,7 & 31,5 & 16,8 & 7,3 \\
\hline \multicolumn{6}{|c|}{ Informação sobre alimentação ** } \\
\hline Sim & 199 & 53,8 & 32,2 & 12,6 & 4,0 \\
\hline Não & 171 & 46,2 & 34,5 & 24,0 & 10,5 \\
\hline \multicolumn{6}{|l|}{ Morbidades } \\
\hline \multicolumn{6}{|l|}{ Diarreia * } \\
\hline Sim & 41 & 11,1 & 24,4 & 34,1 & 14,6 \\
\hline Não & 329 & 88,9 & 34,3 & 15,2 & 6,1 \\
\hline \multicolumn{6}{|l|}{ Febre e tosse * } \\
\hline Sim & 50 & 13,6 & 32,0 & 32,0 & 12,0 \\
\hline Não & 319 & 86,4 & 33,5 & 15,7 & 6,3 \\
\hline \multicolumn{6}{|l|}{ Sibilância } \\
\hline $\operatorname{Sim}$ & 166 & 45,1 & 33,7 & 21,7 & 8,4 \\
\hline Não & 202 & 54,9 & 33,2 & 14,9 & 5,4 \\
\hline \multicolumn{6}{|c|}{ ESF = Estratégia Saúde da Família; ACS= Agente Comunitário de Saúde. } \\
\hline
\end{tabular}


Tabela 2. Análise bivariada, OR para insegurança alimentar segundo assistência em saúde e morbidades infantis, Crato(CE), Brasil, 2010.

\begin{tabular}{lccc}
\hline Variáveis & Odds Ratio & (IC95\%) & $\boldsymbol{p}$ \\
\hline Assistência em saúde & & & \\
Atendido pela ESF & 2,54 & $1,66-3,88$ & $<0,0001$ \\
Visita do ACS & 1,74 & $1,12-2,62$ & 0,013 \\
2 - -30 trimestre de início do pré-natal & 3,21 & $1,43-7,18$ & 0,003 \\
$<6$ consultas de pré-natal & 2,55 & $1,20-5,39$ & 0,012 \\
Falta de informação sobre alimentação & 2,34 & $1,53-3,59$ & $<0,0001$ \\
Morbidades & & \\
Diarreia & 2,12 & 0,038 \\
Febre e tosse & 2,54 & $1,03-4,38$ & 0,006 \\
Sibilância & 1,54 & $1,28-5,04$ & 0,044 \\
\hline IC = Intervalo de Confiança; ESF = Estratégia Saúde da Família; ACS= Agente Comunitário de Saúde. & $1,01-2,34$ \\
\end{tabular}

A Tabela 1 apresenta a análise univariada dos níveis de IA em relação à assistência de saúde e morbidades. Podemos destacar que as familias atendidas pela Estratégia Saúde da Família (ESF), com mulheres que iniciaram o pré-natal entre o 2 o e 30 trimestre de gravidez e que não receberam informação sobre alimentação infantil apresentaram, na Tabela 2, valores de IA cerca de duas vezes maiores que as familias não atendidas pela ESF, com mulheres que iniciaram o pré-natal no 1o trimestre e que receberam informações sobre alimentação infantil $(p<0,05)$.

Observamos também nesta população que pouco mais da metade dos entrevistados (53,8\%) referiu ter recebido informações sobre alimentação da criança, sendo o médico o profissional que mais efetuou tal orientação $(55,8 \%)$ e a principal forma foi à conversa durante a consulta $(71,4 \%)$. Neste aspecto, as informações mais citadas foram relacionadas à alimentação infantil (34,1\%) como horário, consistência e higiene; ao aleitamento materno exclusivo $(19,4 \%)$ e ao consumo de frutas, verduras e legumes $(15,2 \%)$. Informações pouco mencionadas foram: transição alimentar entre quatro e seis meses de idade $(7,4 \%)$ e alimentação para morbidades específicas (4,1\%). Além disso, 19,8\% dos entrevistados não lembravam qual a principal informação.

Quanto aos eventos de morbidade apresentados pelas ocorrências de diarreia, febre e tosse nos últimos 15 dias, esses mostraram-se associados com insegurança alimentar no nível mais grave $(\phi<0,05)$. Por sua vez, não foi encontrada associação significativa entre IA e a suplementação por Vitamina A ou Ferro, segundo o teste Qui-quadrado $(p>0,05)$. Quanto às ações voltadas à saúde da criança: 63,1\% receberam suplementação de Vitamina A nos últimos seis meses. Sobre o combate à anemia, apenas $35,6 \%$ das crianças receberam sulfato ferroso.

$\mathrm{Na}$ Tabela 2 onde são apresentados os odds ratio para insegurança alimentar, nota-se que maiores chances de situação de IA $(<0,01)$ encontram-se em famílias atendidas pela ESF, mães que não completaram o pré-natal e ausência de orientação nutricional. Além disso, em situação de IA crianças tiveram 2,5 mais chances de apresentar febre e tosse nos últimos 15 dias.

\section{DISCUSSÃO}

As famílias em situação de IA no presente estudo foram, em sua maioria, assistidas pela Estratégia Saúde da Família (ESF). Sabe-se que dentre os indicadores socioeconômicos, a renda é a variável que mais influencia a insegurança alimentar [9], principalmente nas famílias do nordeste brasileiro [11,12]. Assim, esse achado pode ser explicado pelo fato de que as famílias mais vulneráveis, de baixa renda e em IA são as que têm acesso ao programa de promoção da saúde, ESF, uma vez que esta assistência é uma estratégia de oferecer às famílias atenção à saúde preventiva e curativa, em suas próprias comunidades e desenvolver atividades educativas desde o período prénatal, na busca de garantir uma assistência maternoinfantil mais efetiva [13]. 
Quanto às prevalências de morbidades, as crianças menores de cinco anos que apresentaram diarreia, sibilância (chiado no peito), febre e tosse foram superiores a média nacional $(9,4 \%, 34 \%$ e $13 \%$, respectivamente), segundo o PNDS-2006 [9]. Pode-se supor que os assuntos abordados sobre alimentação na ESF não foram bem assimilados pelos responsáveis das crianças. Visto que informações sobre a higiene dos alimentos, que foram as mais citadas pelos entrevistados, não apresentam concordância com o percentual elevado de casos de diarreia em crianças nos últimos 15 dias.

Contudo, são as infecções respiratórias agudas, representadas pela ocorrência de febre e tosse, que apareceram como a principal morbidade associada à situação de IA. Mesmo representando pequena prevalência, essas constituem a principal causa de morte em crianças menores de cinco anos de idade [14,15]. A associação entre IA e problemas de saúde encontrada nesta pesquisa é semelhante aos resultados de outra população que apresenta crianças menores de 36 meses de idade em IA, que tiveram quase duas vezes mais chances de terem a saúde "ruim ou muito ruim", com probabilidade quase $1 / 3$ maior de serem hospitalizadas desde o nascimento [16]. Somando-se a isso Hackett, Melgar-Quiñonez e Alvarez [17] encontraram em crianças pré-escolares da Colômbia associação significativa de IA com infecções respiratórias, além de episódios de diarreia e diagnóstico de parasitoses $(p<0,0001)$. Dentro desta perspectiva, pode-se deduzir que crianças pequenas expostas à situação de IA têm alta vulnerabilidade à doença, estando em coerência com os nossos achados.

Quanto ao pré-natal, à realização de mais de seis consultas e o início do atendimento no primeiro trimestre foram indicadores de segurança alimentar, em sintonia com resultados diretos dos grandes progressos brasileiros, evidenciados na última década em relação ao acesso de mulheres ao acompanhamento gestacional, ações também realizadas pela ESF [?].

Desta forma, evidências reforçam que $\mathrm{O}$ número de mulheres grávidas que não se submetem a nenhuma consulta pré-natal vem diminuindo. Também houve um salto importante no percentual de mulheres que passaram a realizar a primeira consulta pré-natal nos três primeiros meses de gestação, principalmente no Nordeste [9], resultados que vêm sendo apontados desde a Chamada Nutricional para o semiárido ${ }^{[18]}$ como também para o Ceará ${ }^{19]}$. Assim, o maior acesso aos serviços de saúde e assistência materna são avanços relacionados à implementação de programas que resultaram em significativa melhoria de vida de mulheres e crianças, tanto nacional como regional.

Entretanto, não foi observado tal avanço em relação aos programas de suplementação de micronutrientes, na medida em que se pode considerar insatisfatório o recebimento de Vitamina A e principalmente do suplemento de ferro. É possível que as baixas coberturas dessas intervenções possam ser determinadas pelo pouco conhecimento dos responsáveis pelas crianças, como também dos profissionais e gestores sobre sua relevância ${ }^{[20]}$.

Além disso, observamos a ausência de informações sobre a alimentação infantil entre famílias em IA. Já que o assunto transição alimentar na infância foi muito pouco citado, inclusive entre as famílias em $\mathrm{SAN}$, mesmo sendo este considerado atualmente o aspecto mais importante devido ao aumento da obesidade ${ }^{[9]}$, principalmente nessa população, que apresenta acentuado percentual de sobrepeso de acordo com o indicador peso/altura (16,8\%) [21].

De acordo com Souza [22], a insuficiência das ações específicas de Educação Alimentar e Nutricional (EAN) voltado para a redução da obesidade, visando mudança no hábito de consumo, deve-se ao orçamento desigual do Plano Plurianual (2012-2015) [23] que prioriza programas de curto prazo (emergenciais) do que programas de longo prazo (estruturais), limitando os avanços na política de SAN. Dessa forma, percebe-se a necessidade da capacitação de profissionais de saúde, como observados em outros estudos [24,25], para viabilizar a integralidade das ações dos programas nacionais de SAN, principalmente voltados para a população socioeconomicamente desfavorável.

Todavia, entre os achados obtidos, podemos discutir um aspecto positivo da diferença de 10\% a mais de atendimento pelo ACS em comparação a ESF, o que pode referir ao reconhecimento desses facilitadores pela comunidade, por serem mais próximos e atuantes no cerne familiar por suas visitas domiciliares. Esse achado demonstra que o ACS aproxima-se mais da população do que o conceito da ESF, lembrando que o relato da visita do ACS significa que ESF está atuando na área, mesmo não sendo reconhecida como forma de atendimento por esses $10 \%$ de famílias. Possivelmente, o ACS é a figura 
espontaneamente mais ligada a essas famílias, como comentado anteriormente, e esse acesso direto e constante com a comunidade pode melhorar significativamente a divulgação dos direitos à segurança alimentar e nutricional.

Portanto, o ACS é o profissional da saúde que mais possibilita a EAN como estratégia à promoção a práticas alimentares saudáveis, devido a sua realidade similar e a relação de confiança com a população local, permitindo o reconhecimento sobre o DHAA no campo da saúde comunitária ${ }^{[26] .}$

Mesmo com as conquistas do governo brasileiro na área socioeconômica ou em termos dos indicadores de saúde e nutrição observa-se dificuldades aos programas ligados ao abastecimento, educação alimentar e nutricional e agricultura familiar, uma vez que ao longo dos anos por problemas na execução ou exclusivismo do Programa Bolsa Família dificultaram o funcionamento de um sistema integrado de SAN [27]. Deste modo, há a necessidade de qualificação em prática educativa alimentar e nutricional do ACS, a partir da educação popular na ampliação da alimentação saudável e da saúde em sua área de trabalho, favorecendo o desenvolvimento do exercício crítico e político do sujeito, inclusão social, melhoria da qualidade de vida ${ }^{[26]}$.

\section{CONCLUSÃO}

Com base no presente estudo, a Estratégia Saúde da Família deve ser aprimorada, com profissionais de saúde, principalmente Agentes Comunitários de Saúde, mais capacitados para liderar ações educativas em relação à alimentação de crianças em transição de faixa etária, o que pode reduzir a incidência de patologias agudas como diarréia, febre e tosse, além de doenças crônicas, como a obesidade infantil.

Cabe aqui ressaltar a necessidade de formulação e implementação de Políticas Públicas de Saúde para maior interação da atenção à saúde com determinantes sociais, econômicos, políticos, possibilitando o monitoramento das condições de vida e bem estar da população. Portanto, o governo e a sociedade civil devem ter maior atenção sobre os programas de Educação Alimentar e Nutricional, viabilizando o entendimento de alimentação saudável como um direito universal.

\section{AGRADECIMENTO}

Agradecemos ao Professor Paulo César de Almeida pela amostragem e suporte estatístico; à Secretaria de Saúde Municipal do Crato; ao Laboratório de Segurança Alimentar e Nutricional da Universidade Estadual do Ceará (UECE); aos acadêmicos voluntários da Universidade Regional do Cariri; à Coordenação de Aperfeiçoamento de Pessoal de Nível Superior (CAPES), pela bolsa de estudo, vinculada ao Programa de Pós-graduação em Ciências da Nutrição da Universidade Federal da Paraíba.

\section{REFERÊNCIAS}

[1] Valente FLS. Fome, desnutrição e cidadania: inclusão social e direitos humanos. Saúde Soc. 2003;12(1):51-60.

[2] Freitas MCS, Pena PGL. Segurança alimentar e nutricional: a produção do conhecimento com ênfase nos aspectos da cultura. Rev Nutr. 2007;20(1):69-81.

[3] Habicht JP, Pelto G, Frongillo E, Rose D. Conceptualization and instrumentation of food insecurity. In: National Academy of Sciences Workshop - Cornell University - Tulane University. Tulane; 2004.

[4] Malta DC, Medeiros NS, Accioly MC, Bonollo P, Aranha A, Goulart EMA et al. Inquérito nutricional em crianças menores de cinco anos de Belo Horizonte em 1993. Rev Med Minas Gerais. 1998;8(4):141-144.

[5] Batista-Filho M, Ferreira LOC. Um modelo para avaliação rápida da situação nutricional e de saúde de crianças e mães no dia nacional de vacinação. Rev. Bras. Saúde Mater. Infant. 2001;1(2):145-154.

[6] Brasil. Ministério da Saúde. Prevalência de aleitamento materno nas capitais brasileiras e no Distrito Federal: relatório final. Brasília; 2000.

[7] Brasil. Sistema de Informação do Programa Nacional de Imunizações. DATASUS. Campanha Nacional de vacinação contra polio 2009, Ceará [internet]. [acesso em 10 maio 2010]. Disponível em http://pni.datasus.gov.br

[8] IBGE - Instituto Brasileiro de Geografia e Estatística. Pesquisa Nacional por Amostra de Domicílios - PNAD: suplemento sobre segurança alimentar 2004. Rio de Janeiro; 2006.

[9] Brasil. Ministério da Saúde. Centro Brasileiro de Análise e Planejamento. Pesquisa Nacional de Demografia e Saúde da 
Criança e da Mulher - PNDS 2006: dimensões do processo reprodutivo e da saúde da criança. Brasília; 2009.

[10] Segall-Corrêa AM, Pérez-Escamilla R, Maranha LK, Sampaio MFA, Yuyama L, Alencar F, et al. Projeto: acompanhamento e avaliação da segurança alimentar de famílias brasileiras: validação de metodologia e de instrumento de coleta de informação - Relatório Técnico. Campinas; 2004.

[11] Oliveira JS, Lira PIC, Andrade SLLS, Sales AC, Maia SR, Batista-Filho MB. Insegurança Alimentar e estado nutricional de crianças de São João do Tigre, no semi-árido do Nordeste. Rev Bras Epidemiol. 2009;12(3):413-423.

[12] Oliveira JS, Lira PIC, Maia SR, Siqueira LAS, Amorim RCA, Batista-Filho MB. Insegurança alimentar e estado nutricional de crianças de Gameleira, zona da mata do Nordeste brasileiro. Rev Bras Saúde Mater Infant. 2010;10(2):237-245.

[13] Parada CMGL, Carvalhaes MABL, Winckler CC, Winckler LA, Winckler VC. Situação do aleitamento materno em população assistida pelo programa de saúde da família - PSF. Rev. Latino-Am Enfermagem. 2005;13(3):407-414.

[14] Mulholland K. Childhood pneumonia mortality - a permanent global emergency. Lancet. 2007;370(9583):285289.

[15] Rudan I, Boschi-Pinto C, Biloglav Z, Mulholland K, Campbell H. Epidemiology and etiology of childhood pneumonia. Bull World Health Organ. 2008;86(5):408-416.

[16] Cook JT, Frank DA, Berkowitz C, Black MM, Casey $\mathrm{PH}$, Cutts DB, et al. Food Insecurity is Associated with Adverse Health Outcomes among Human Infants and Toddlers. J Nutr. 2004;134(6):1432-38.

[17] Hackett M, Melgar-Quiñonez H, Álvarez MC. Household food insecurity associated with stunting and underweight among preschool children in Antioquia, Colombia. Rev Panam Salud Publica. 2009;25(6):506-510.

[18] Monteiro CA, Conde WL, Konno SC. Análise do inquérito chamada nutricional 2005. Cad Estud Desenv Soc Debate. 2006;4:29-36.

[19] Diniz DB, Fernandes DLA, Mamede ALES. Estado nutricional de crianças menores de cinco anos do semi-árido do Ceará. Cad Estud Desenv Soc Debate. 2006;4:51-56.
[20] Almeida ER, Carvalho AT, Nilson EAF, Coutinho JG, Ubarana JA. Avaliação participativa do Programa Nacional de Suplementação de Vitamina A em um município da Região Nordeste do Brasil. Cad Saúde Pública 2010;26(5):949-960.

[21] Rocha EMB, Lima RT, Diniz DB, Almeida PC. Situação nutricional de crianças em município de privilegiado Índice de Desenvolvimento Humano do semiárido brasileiro e sua relação com Insegurança Alimentar. Segurança Alimentar e Nutricional. 2012;19(2):17-29.

[22] Souza LR. Avaliação de Políticas e Programas de Segurança Alimentar e Nutricional no Brasil: uma análise a partir do Plano Plurianual (PAA 2012-2015). Segurança Alimentar e Nutricional. 2013;20(Suppl):182-193.

[23] Brasil. Plano Plurianual 2012-2015 (PPA 2012-2015). Relatório de comunicação mista de planos, orçamentos públicos e fiscalização do Congresso Nacional (CMO) alterações do Plano Plurianual 2012-2015 [internet]. [acesso em 20 jul 2013]. Disponível em http://www.planejamento.gov.br/secretarias/upload/Arqui vos/spi/PPA/2012/Atualizacao_do_PPA_2012_2015.pdf

[24] Barroso GS, Sichieri R, Salles-Costa R. Fatores associados ao déficit nutricional em crianças residentes em uma área de prevalência elevada de insegurança alimentar. Rev Bras Epidemiol. 2008;11(3):484-494.

[25] Pereira JA, Paiva AA, Bergamaschi DP, Rondó PHC, Oliveira GC, Lopes IBM, et al. Concentrações de retinol e de beta-caroteno séricos e perfil nutricional de crianças em Teresina, Piauí, Brasil. Rev Bras Epidemiol. 2008;11(2):287296.

[26] Amparo-Santos L, Soares MD, Magalhães LM, Reis ABC, Porcino DC, Santos MO, et al. Segurança Alimentar e Nutricional e Tecnologias Sociais em Educação Alimentar e Nutricional: notas sobre um projeto de pesquisa e extensão. Segurança Alimentar e Nutricional. 2013;20(Suppl):156-168.

[27] Belik W. A Política Brasileira de Segurança Alimentar e Nutricional: concepção e resultados. Segurança Alimentar e Nutricional. 2012;19(2):94-110. 